\title{
Peritoneal lymphomatosis mimicking peritoneal carcinomatosis: important imaging clues for correct diagnosis
}

David Soon Yiew Sia ${ }^{1}$, MBBS, FRCR, Jeevesh $\underline{K a p u r}^{1}$, MBBS, FRCR, Yee Liang $\underline{T h i a n}^{1}$, MBBS, FRCR

\begin{abstract}
Peritoneal lymphomatosis, a rare presentation of lymphoma, can mimic peritoneal carcinomatosis. Computed tomography findings in both conditions include omental caking, as well as peritoneal enhancement, thickening and nodularity. We report three cases of peritoneal lymphomatosis and give an analysis of the salient imaging features that aided in the accurate diagnosis of the condition in each case. Two of the three cases demonstrated elevated serum cancer antigen 125 levels, a marker commonly associated with ovarian carcinoma. It is thus critical to distinguish peritoneal lymphomatosis from carcinomatosis, as the prognosis and management are dramatically different.
\end{abstract}

Keywords: CA 125, computed tomography, magnetic resonance imaging, peritoneal lymphomatosis, uterine lymphoma

\section{INTRODUCTION}

The majority of primary cancers that spread to the peritoneum are carcinomas that commonly originate from the ovary, gastrointestinal tract and breast. Metastases to the peritoneal space manifest clinically as abdominal swelling and discomfort. Such symptoms are frequently the first presentation of malignancy. Although lymphomatous infiltration of the peritoneum is not common, early diagnosis is crucial in order to avoid unnecessary investigations for a primary carcinoma and the need to drastically alter the prognosis and management of the disease. The crosssectional imaging findings of peritoneal lymphomatosis (e.g. peritoneal enhancement, thickening, nodularity and ascites with omental caking) are almost indistinguishable from that of peritoneal carcinomatosis. We report three cases of peritoneal lymphomatosis, with two exhibiting raised serum cancer antigen (CA) 125 levels, which further confounded the diagnosis. We also discuss additional imaging features that are highly atypical of peritoneal carcinomatosis, which aid in the diagnosis of peritoneal lymphomatosis.

\section{CASE REPORTS}

\section{Case 1}

A 68-year-old Chinese Indonesian woman presented with abdominal discomfort and distension that had persisted for one month. Physical examination found an enlarged uterus (10-12 weeks in size) and moderate ascites. Ultrasonography (US) of the abdomen confirmed the presence of uterine enlargement. Chest radiography revealed moderate right pleural effusion and an elevated serum CA 125 level of $345 \mathrm{U} / \mathrm{mL}$ (normal $<35 \mathrm{U} / \mathrm{mL}$ ). Based on initial investigations, the working diagnosis was primary uterine/ovarian carcinoma with peritoneal dissemination. An urgent referral was made to the Obstetrics and
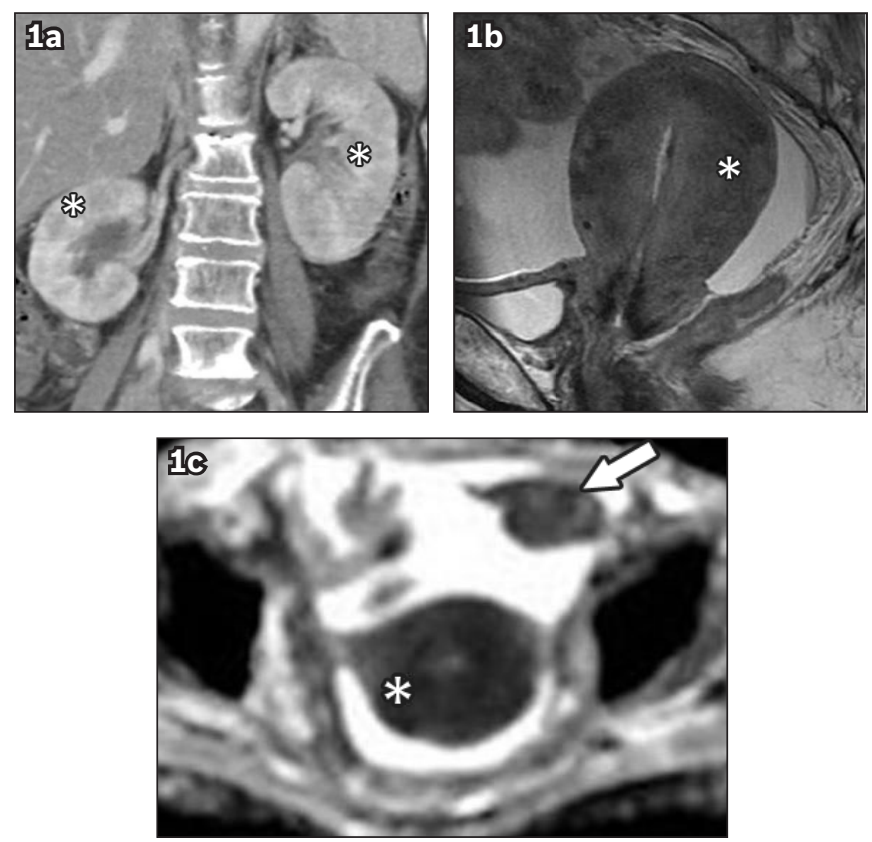

Fig. 1 (a) Coronal CT image shows enlargement of both kidneys $\left({ }^{*}\right)$. Although the kidneys displayed a heterogeneous enhancement pattern, there was preservation of the kidneys' outlines. (b) Sagittal T2-W MR image shows a diffusely enlarged uterus $\left(^{*}\right)$ with obliteration of the junctional zone and replacement of the myometrium by an infiltrative T2 hyperintense lesion and surrounding ascites. The zonal anatomy of the cervix is preserved. (c) Axial apparent diffusion coefficient $(A D C)$ map of the uterus ( $b$-value $=0-1,000 \mathrm{~s} / \mathrm{mm}^{2}$ ) shows severely restricted diffusion of the myometrium (*) with a mean ADC value of $0.8 \times 10^{-3} \mathrm{~mm}^{2} / \mathrm{s}$. This indicates dense cellularity, which is characteristic of lymphomatous infiltration. Peritoneal deposit (arrow) with similar restricted diffusion is also seen.

Gynaecology Department. However, colposcopy and Pap smear were normal, and endometrial sampling was inconclusive due to inadequate tissue yield. Further evaluation with computed tomography (CT) of the abdomen and pelvis revealed moderate

${ }^{1}$ Department of Diagnostic Imaging, National University Hospital, Singapore

Correspondence: Dr David Sia Soon Yiew, Registrar, Department of Diagnostic Imaging, National University Hospital, 5 Lower Kent Ridge Road, Singapore 119074. davidssy@yahoo.com 
ascites with omental caking, and marked peritoneal thickening, nodularity and enhancement. Bilateral pleural effusions were also present. While the patient's kidneys displayed a heterogeneous pattern of contrast enhancement, their reniform shapes were preserved (Fig. 1a). Magnetic resonance (MR) imaging of the pelvis showed a diffusely enlarged uterus, with obliteration of the junctional zone by an infiltrative and ill-defined lesion (Fig. 1b). The lesion was hyperintense on T2-weighted images. There was also marked globally restricted diffusion of the uterus (Fig. 1c). The patient's cervix and endometrium were preserved and no adnexal masses were seen. US-guided pleural drainage was performed due to the patient's worsening dyspnoea. Pleural fluid cytological analysis confirmed the diagnosis of B-cell non-Hodgkin's lymphoma. However, the patient was not amenable to imaging-guided biopsy due to the absence of a sufficiently large mass or lymph node. Open surgical biopsy was offered, but was declined by the patient due to financial constraints. She opted to seek treatment in her own country.

\section{Case 2}

An 86-year-old Chinese woman, with a significant medical history of hyperthyroidism and hypertension, presented with abdominal discomfort that had persisted for two weeks. Physical examination did not reveal any abdominal mass. Tumour marker screening showed an elevated serum CA 125 level of $366 \mathrm{U} / \mathrm{mL}$, but carcinoembryonic antigen and alpha foetoprotein levels were normal. Chest radiography revealed multiple subcentimetre pulmonary nodules and bilateral pleural effusions. CT of the abdomen and pelvis showed widespread soft tissue nodularity, stranding of the omentum and ascites. The patient's spleen was enlarged and deformed by multiple hypodense nodules, while the liver appeared normal without any evidence of intrahepatic nodules. There were also multiple enlarged mesenteric and retroperitoneal lymph nodes (Fig. 2a). CT of the thorax showed extensive mediastinal and bilateral hilar lymphadenopathy, in addition to right pleural effusion and multiple, bilateral pulmonary and pleural nodules (Fig. 2b). USguided right pleural drainage was performed for symptomatic relief, and pleural fluid cytological analysis revealed B-cell non-Hodgkin's lymphoma. No diagnostic peritoneal tap was performed. The patient was started on rituximab, followed by bendamustine five days later. However, she developed type 2 respiratory failure secondary to hospital-acquired pneumonia, requiring intensive care unit management. Her condition deteriorated and she succumbed to the disease two weeks later.

\section{Case 3}

A 41-year-old Chinese man presented with loss of appetite and weight, and abdominal distension that had persisted for two weeks. On gastroscopy, a large tumour with extensive ulceration was found along the lesser curve of the stomach, and hence, a biopsy was performed. CT of the abdomen further demonstrated
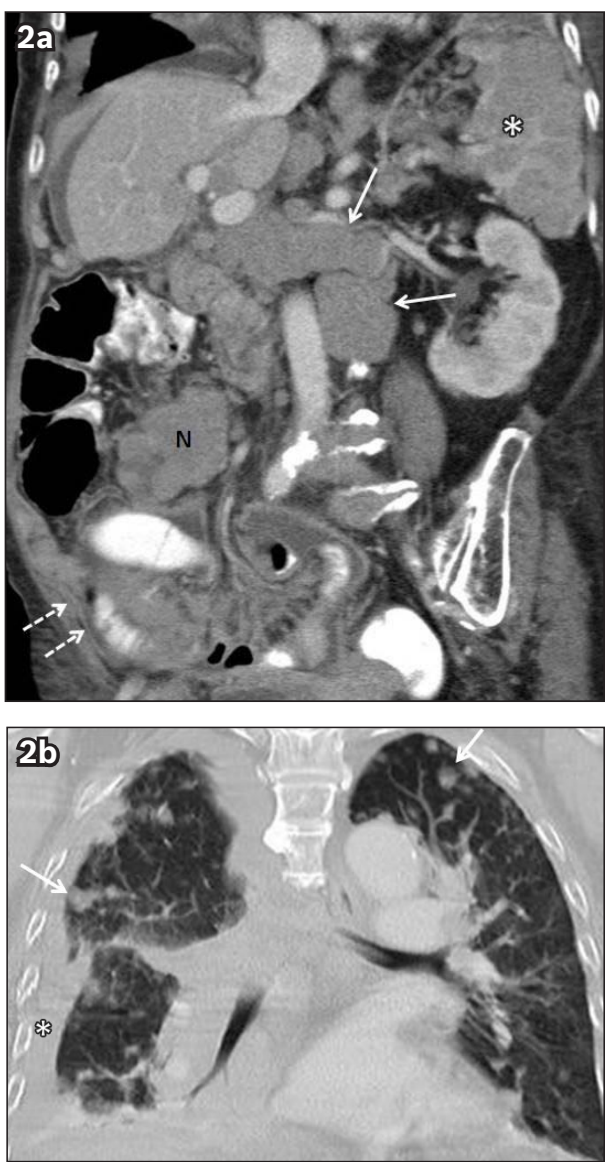

Fig. 2 (a) Coronal CT image shows multiple hypoattenuating splenic nodules $\left({ }^{*}\right)$, retroperitoneal adenopathy (arrows), mesenteric confluent nodules $(\mathrm{N})$ and peritoneal thickening (broken arrows). These findings do not distinguish peritoneal lymphomatosis from peritoneal carcinomatosis or tuberculosis (b) Coronal CT image shows multiple pulmonary nodules (arrows) with right pleural effusion $\left({ }^{*}\right)$.

the extent of the gastric tumour. There was a large volume of ascites with omental caking, as well as peritoneal and mesenteric soft tissue deposits. Additionally, extensive segments of the jejunum showed circumferential thickening with preserved luminal patency, giving rise to a pipe-like appearance (Fig. 3). The patient's retroperitoneal lymph nodes were not enlarged. Histopathological diagnosis of the endoscopic tissue sample revealed aggressive, large $B$-cell lymphoma, with positive staining for CD20, CD79A and CD10. The patient was started on CHOP (cyclophosphamide, hydroxydaunorubicin, oncovin and prednisone) chemotherapy regime one week after presentation.

\section{DISCUSSION}

Lymphoma, known to be a great mimicker, is a common haematological malignancy that can involve any part of the body. ${ }^{(1)}$ However, diffuse infiltration of the peritoneum by lymphoma is rare. Although the exact incidence of peritoneal lymphomatosis has not been well established, one series of 107 cytology-proven cases of malignant ascites reported 10 (9\%) cases of lymphoma, with ascites as the primary complaint in six patients. $^{(2)}$ 


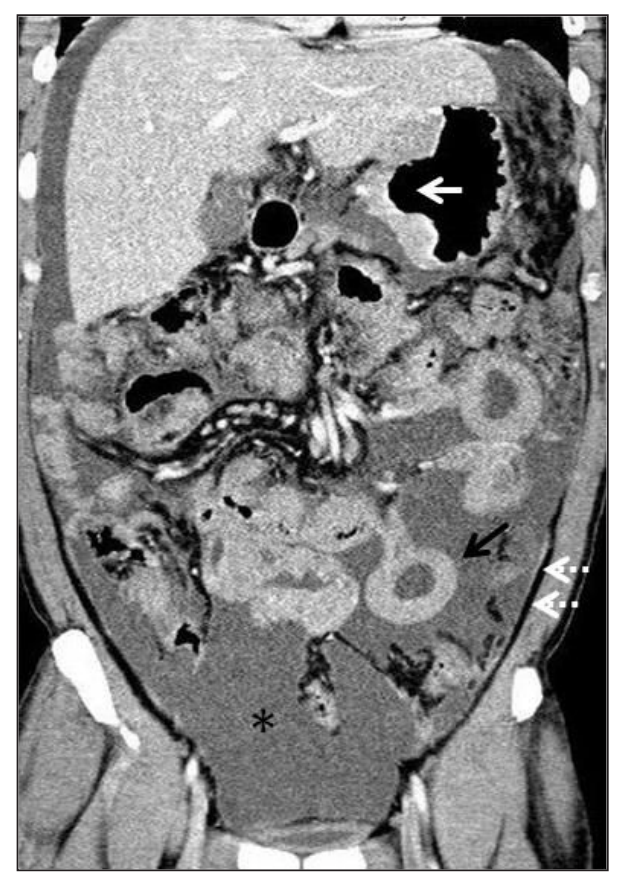

Fig. 3 Coronal CT image shows a large ulcerative gastric mass (white arrow) that is highly suspicious for primary gastric adenocarcinoma. However, the presence of diffuse, smooth circumferential thickening and dilatation of the long segments of the small bowel (black arrow) is unusual for peritoneal carcinomatosis, and more typically seen in lymphoma. Note gross ascites $\left({ }^{*}\right)$ with peritoneal enhancement (white broken arrows)

Peritoneal lymphomatosis may occur as a secondary involvement from a visceral lymphoma, or as a primary lymphoma of the peritoneum. Even though the literature offers no direct comparison of the incidences of these two entities, peritoneal lymphomatosis with visceral involvement is generally much more common than that with primary peritoneal lymphoma involvement. In one autopsy series, 64 (20\%) of the 322 patients with disseminated lymphoma were found to have peritoneal and omental disease. (3) Primary peritoneal lymphoma without visceral involvement is known as body cavity lymphoma or primary effusion lymphoma. It is seen almost exclusively in patients with human immunodeficiency virus (HIV), and has been estimated to account for $4 \%$ of all HIV-associated lymphomas. ${ }^{(4)}$

The imaging features of peritoneal lymphomatosis that are highly indistinguishable from peritoneal carcinomatosis include omental caking as well as peritoneal and mesenteric soft tissue nodularity and enhancement, with or without ascites. Mesenteric and retroperitoneal lymphadenopathies are frequently seen, but the degree of adenopathy may not be specific enough to distinguish the lymphomas from nodal metastases that arise from other tumours. In recent years, there have been case reports of diagnostic confusion resulting in false presumptive diagnoses, which led to unnecessary exploratory laparotomy and a delay in chemotherapy commencement. ${ }^{(7,8)}$ This could have potentially been avoided if lymphoma was included in the differential diagnosis of peritoneal disease. The three cases presented in our report have concomitant imaging findings in the abdominal and pelvic viscera that strongly suggest the presence of lymphoma. Awareness of these imaging clues will expedite the correct diagnosis of lymphomas and thus avoid unnecessary and frequently invasive investigations for a primary tumour.

In Case 1, lymphomatous infiltration of the uterus led to global uterine enlargement, which was seen on CT. The smooth contour of the patient's uterus was uniquely preserved without the identification of any discrete focal lesions. Infiltration and enlargement of both kidneys resulted in a heterogeneous appearance of the renal parenchyma on imaging, but the bean shape was maintained. These imaging findings are typical of organ-infiltrative manifestations of lymphoma and are not seen in peritoneal carcinomatosis, whether in cases of primary peritoneal malignancy arising from the mesothelium or cases of transcoelomic dissemination of tumour. Another clue obtained on MR imaging was the restricted diffusion of the patient's entire uterine myometrium. This indicated extremely dense cellularity, which is compatible with neoplastic infiltration.

Lymphomatous involvement of the uterus in disseminated disease is much more common than primary uterine lymphoma. Although reports of radiological findings of uterine lymphoma are limited, diffuse enlargement of the uterus with relatively homogeneous MR imaging signal irrespective of tumour size, diffuse uterine enlargement without disruption of endometrial and cervical epithelia, and extensive involvement of the uterine body with preservation of normal zonal anatomy of the uterine cervix, have been described. ${ }^{(9-11)}$ Our findings in Case 1 are similar to those of Kawakami et al and Suzuki et al. ${ }^{(10,11)}$

Renal lymphoma commonly presents as part of a disseminated multisystemic disease and rarely occurs in isolation. On CT imaging, it can appear as solitary or multiple renal masses, direct spread from a retroperitoneal mass, perirenal lesions that are separate from renal parenchyma, or diffuse renal parenchymal infiltration. Infiltration within the interstitium of the kidney replaces the normal parenchyma and manifests as nephromegaly, with the normal renal contour retained. This pattern of involvement usually affects both kidneys in approximately $20 \%$ of patients. ${ }^{(12)}$

In Case 2, the CT image showed multiple, rounded nodular masses with hypodense centres in the spleen, as well as widespread omental caking. Such findings should alert the radiologist of the possibility of lymphoma, as it is the most common primary malignancy of the spleen. ${ }^{(1)}$ Splenic involvement is seen in $20 \%-40 \%$ of patients with lymphoma. ${ }^{(13)}$ In these cases, the spleen may demonstrate focal nodules or diffuse enlargement. The nodules are typically hypoattenuating, with reduced contrast material enhancement compared to normal splenic tissue. Focal splenic lymphomas can be indistinguishable from metastases, although the former are usually smaller, appear homogeneous and occur with enlarged coalescent lymph nodes. ${ }^{(13)}$ Although metastatic gastrointestinal adenocarcinoma is still a diagnostic possibility, it would be highly unusual for splenic metastases to occur in the absence of hepatic metastases 
due to the preferential drainage of the gastrointestinal tract into the portal venous system.

Due to the high prevalence of disseminated tuberculosis (TB) in the Asian population, disseminated TB is an important differential diagnosis of peritoneal disease, particularly when the spleen and/or lungs are involved. Many radiological features of abdominal TB such as ascites, mesenteric and omental thickening, and lymphadenopathy with hypoattenuating centres, have been described. However, these features cannot be reliably distinguished from those arising from malignant causes. To differentiate tuberculous peritonitis from malignant peritoneal disease, a combination of acid-fast bacillus (AFB) staining of sputum, pleural or peritoneal fluid, TB molecular test and tissue biopsy should be done.

Case 3 presented with a large gastric mass and ascites with peritoneal nodularity - findings that are typically suggestive of metastatic gastric cancer. However, the morphology of the small bowel on CT was an imaging clue for correct diagnosis. It showed smooth and uniform pipe-like thickening of a long continuous segment of the small bowel with homogeneous attenuation. This is characteristic of lymphoma. The observed morphology is different from that of peritoneal carcinomatosis, where the involved small bowel is typically irregularly and asymmetrically thickened, with heterogeneous attenuation due to serosal tumour deposits. In lymphomas, attenuation of the small bowel is homogeneous, as the process is more infiltrative.

The small bowel is the second most common site of gastrointestinal lymphoma. ${ }^{(14)}$ Focal or diffuse symmetrical thickening of the involved segment occurs as a result of tumour extension along the submucosa and muscularis propria. Characteristically, there is no significant stenosis despite the degree of mural thickening. This infiltrative process ultimately results in a dilated pipe-like segment of the bowel. Peristalsis may be impaired secondary to destruction of the autonomic plexus, resulting in a non-peristaltic, aneurysmally dilated segment. ${ }^{(15)}$

Despite the presence of important imaging clues that favour the diagnosis of lymphoma, definitive histological confirmation is still required. A major limitation of Cases 1 and 2 was the lack of direct histological proof of visceral lymphomatous infiltration. However, the imaging findings are characteristic for extranodal lymphoma, and this is strongly supported by the pleural fluid cytology. The emphasis on radiological examinations is to provide crucial leads that aid the clinician in carrying out a more targeted investigation and biopsy. In order to confirm the diagnosis as well as acquire vital information (e.g. grade and immunophenotypic characteristics) to guide further treatment, tissue samples for histopathologic examination need to be obtained either by imaging-guided or open surgical biopsy.

Markedly elevated serum CA 125 levels, as found in Cases 1 and 2, can lead to further diagnostic confusion as this tumour marker is most commonly associated with ovarian cancer. Horger et al reported similar diagnostic confusion resulting from elevated serum CA 125 level and the presence of pelvic masses. ${ }^{(7)}$ CA 125 is a glycoprotein secreted by normal coelomic epithelium, such as the peritoneum and pleura. Hence, it can be elevated in benign and malignant conditions that involve the coelomic epithelium. Several investigators have reported serum elevations of CA 125 in up to $40 \%$ of patients with non-Hodgkin's lymphoma. ${ }^{(16)}$ In a study by Abd El Gawad et al, CA 125 level was found to correlate with tumour burden, presence of effusion, treatment response and survival in non-Hodgkin's lymphoma. ${ }^{(17)}$ We wish to highlight that even markedly elevated CA 125 levels can be a red herring in the context of peritoneal malignancy, as shown in our three cases.

In conclusion, our three reported cases are an important reminder that peritoneal lymphomatosis can mimic peritoneal carcinomatosis on cross-sectional imaging. Awareness of the characteristic imaging features of lymphomatous visceral organ involvement in the abdomen and knowledge of the association of CA 125 with lymphoma should alert the radiologist and the clinician of the correct diagnosis, and hence, avoid unnecessary investigations and delay in management.

\section{REFERENCES}

1. Thomas AG, Vaidhyanath R, Kirke R, Rajesh A. Extranodal lymphoma from head to toe: part 2, the trunk and extremities. AJR Am J Roentgenol 2011; 197:357-64.

2. Garrison RN, Kaelin LD, Galloway RH, Heuser LS. Malignant ascites. Clinical and experimental observations. Ann Surg 1986; 203:644-51.

3. Lynch MA, Cho KC, Jeffrey RB Jr, Alterman DD, Federle MP. CT of peritoneal lymphomatosis. AJR Am J Roentgenol 1988; 151:713-5.

4. Simonelli $C$, Spina $M$, Cinelli $R$, et al. Clinical features and outcome of primary effusion lymphoma in HIV-infected patients: a single-institution study. J Clin Oncol 2003; 21:3948-54.

5. Karaosmanoglu D, Karcaaltincaba M, Oguz B, et al. CT findings of lymphoma with peritoneal, omental and mesenteric involvement: peritoneal lymphomatosis. Eur J Radiol 2009; 71:313-7.

6. Kim Y, Cho O, Song S, et al. Peritoneal lymphomatosis: CT findings. Abdom Imaging 1998; 23:87-90.

7. Horger M, Müller-Schimpfle M, Yirkin I, Wehrmann M, Claussen CD. Extensive peritoneal and omental lymphomatosis with raised CA 125 mimicking carcinomatosis: CT and intraoperative findings. $\mathrm{Br} \mathrm{J}$ Radiol 2004; 77:71-3.

8. Kim YG, Baek JY, Kim SY, et al. Peritoneal lymphomatosis confounded by prior history of colon cancer: a case report. BMC Cancer 2011; 11:276.

9. Kim YS, Koh BH, Cho OK, Rhim HC. MR imaging of primary uterine lymphoma. Abdom Imaging 1997; 22:441-4.

10. Kawakami S, Togashi K, Kojima N, et al. MR appearance of malignant lymphoma of the uterus. J Comput Assist Tomogr 1995; 19:238-42.

11. Suzuki Y, Tamaki Y, Hasegawa M, Maebayashi K, Mitsuhashi N. Magnetic resonance images of primary malignant lymphoma of the uterine body: a case report. Jpn J Clin Oncol 2000; 30:519-21.

12. Urban BA, Fishman EK. Renal lymphoma: CT patterns with emphasis on helical CT. Radiographics 2000; 20:197-212.

13. Lee WK, Lau EW, Duddalwar VA, Stanley AJ, Ho YY. Abdominal manifestations of extranodal lymphoma: spectrum of imaging findings. AJR Am J Roentgenol 2008; 191:198-206.

14. Cardona DM, Layne A, Lagoo AS. Lymphomas of the gastro-intestinal tract - pathophysiology, pathology, and differential diagnosis. Indian J Pathol Microbiol 2012; 55:1-16.

15. Leite NP, Kased N, Hanna RF, et al. Cross-sectional imaging of extranodal involvement in abdominopelvic lymphoproliferative malignancies. Radiographics 2007; 27:1613-34.

16. Lazzarino M, Orlandi E, Klersy C, et al. Serum CA 125 is of clinical value in the staging and follow-up of patients with non-Hodgkin's lymphoma: correlation with tumour parameters and disease activity. Cancer 1998; 82:576-82.

17. Abd El Gawad IA, Shafik HE. CA 125, a new prognostic marker for aggressive NHL. J Egypt Natl Canc Inst 2009; 21:209-17. 